\title{
Resistance of reduced graphene oxide on polystyrene surface
}

\author{
M. N. Nikolaeva ${ }^{1}$, A. N. Bugrov ${ }^{1,2}$, T. D. Anan'eva ${ }^{1}$, \\ E. V. Gushchina ${ }^{3}$, M. S. Dunaevskii ${ }^{3}$, A. T. Dideikin ${ }^{3}$ \\ ${ }^{1}$ Institute of macromolecular compounds RAS, Bolshoy pr. 31, 199004 St. Petersburg, Russia \\ ${ }^{2}$ Saint Petersburg Electrotechnical University "LETI", \\ ul. Professora Popova 5, 197376 St. Petersburg, Russia \\ ${ }^{3}$ Ioffe Institute, Politekhnicheskaya ul. 26, 194021 St. Petersburg, Russia \\ marianna_n@mail.ru, alexander.n.bugrov@gmail.com, anthracene@hq.macro.ru \\ katgushch@yandex.ru, Mike.Dunaeffsky@mail.ioffe.ru, dideikin@mail.ioffe.ru
}

PACS 36.20.-r; 68.65.Pq; 71.20.Rv; 72.80. Tm; 74.78.-w

DOI 10.17586/2220-8054-2018-9-4-496-499

\begin{abstract}
Reduced graphene oxide flakes of large area, some of which are more than 100 micrometers in diameter, have been produced on polystyrene surface. These flakes were formed during precipitation of composite based on polystyrene with reduced graphene oxide from the benzene by petroleum ether. Extremely low resistances were obtained for these flakes in planar dimension at room temperature. The measured resistance absolute values turned out to be 2 orders of magnitude lower than the resistance of copper. This result is explained by existence of superconducting component in the reduced graphene oxide inclusions.
\end{abstract}

Keywords: reduced graphene oxide, polystyrene, composite, resistance.

Received: 13 April 2018

\section{Introduction}

There are many theoretical and some current experimental studies devoted to the superconductivity of graphene [1-13]. However, until recently, zero or very low resistance of neat graphene has not been fixed. Only manifestations of superconductivity in doped graphene [1,2] or its indirect signs in graphene and graphite have been established. The latter include the external magnetic flux trapping by pyrolytic graphite powder which indicates its superconducting state [3], superconductivity of single-layer graphene deposited on superconductor [4,5], as well as the appearance of Josephson oscillations and Shapiro steps in polymer structures containing reduced graphene oxide (RGO) [12].

It is known that RGO contributes to the appearance of high conductivity $[14,15]$ and superconductivity in sandwich structures when placed between two macroscopic electrodes [12]. In our case, the object of study is the composite based on polystyrene with RGO combining graphene fragments and oxidized groups. In the present work, we revealed the highly conducting state of graphene flakes in planar structures at the room temperature.

It is worth noting that the RGO under consideration was functionalized by 3-(trimethoxysilyl)propyl methacrylate and can participate in radical polymerization with styrene [15]. As a result, covalent bonds between RGO and polystyrene chains are formed, similar to those in work [16]. Previously, it was shown that RGO localization near the surface of the polymer films contributes to high conductivity in the case of styrene copolymerization with functionalized RGO [17]. Beside this, it has been demonstrated by conductive atomic force microscopy method that highly conductive regions of polystyrene/RGO thin films exhibit ohmic current-voltage characteristics [18].

Maximum length dimensions of RGO under consideration revealed by SEM method are only up to $8 \mu \mathrm{m}$ as it was shown in [15]. The chemical precipitation of the RGO composite upon tightening the polymer coils probably promotes the aggregation of the RGO inclusions. Thus, this process can lead to enlargement of areas of RGO inclusions. Thus, the planar RGO conglomerates with sufficient areas for electrical measurements were obtained using the chemical precipitation method of RGO composite from benzene.

\section{Experimental}

Synthetic details for the RGO composite based on polystyrene as well as surface modification technique of natural crystalline graphite can be found in $[14,15,17,19]$. Composite content in the solvents mixture was 5 wt.\%. Benzene/petroleum ether in different ratios, of 1:1, 1:3, 1:2 and 3:2 were used for RGO composite precipitation. The largest areas of graphene inclusions on the polystyrene surface were observed at a 1:1 ratio of benzene/petroleum ether. So, this solvent ratio $(1: 1)$ was chosen for production of sufficient RGO planar structures. Films of the polymer composite were deposited on glass substrate by casting through the dispenser. Scanning 
probe microscope (SPM) Solver P47-Pro (NTMDT) was used for surface roughness measurements. Since RGO flakes were formed on the polystyrene surface, it was possible to evaluate its thickness by SPM. Thicknesses of RGO flakes measured by SPM did not exceed $50 \mathrm{~nm}$. Thus, the objects of our investigation had turned out to be the multilayered RGO flakes obtained in the result of its self-organization during precipitation process.

Areas of obtained RGO flakes were assessed for resistance using the two-probe method. These measurements were planned only to be qualitative relative to copper resistance. Two kinds of electrodes, the diamond SPM tip doped by nitrogen and steel needles with different curvature radii were used. The curvature radii of electrodes were approximately $50 \mathrm{~nm}$ and $15 \mu \mathrm{m}$ correspondingly. Resistance values of RGO flakes were compared with macroscopic copper substrate ones. Pressure on electrodes during measurements did not exceed $0.5 \mathrm{~g} / \mu \mathrm{m}^{2}$. Applying larger pressures caused the breaking of thin SPM electrodes and RGO flakes splitting. After all, at the excess pressure the full separation of RGO flakes from polystyrene surface can be observed.

\section{Results and discussion}

The RGO flake distribution with various shapes and areas on the polystyrene surface after precipitation was irregular and sizes of flakes differed from each other in many times (Fig. 1). This distribution drastically differs from dispersion degree and homogeneity of the filler in the polymer matrix without applying precipitation (Fig. 2). As we can suppose in the precipitation process, polymer coils became tightened. These appearing tensions between RGO planes and polymer coils create conditions for RGO releasing from polymer matrix. In this way, separate RGO particles are getting together into large RGO flakes.

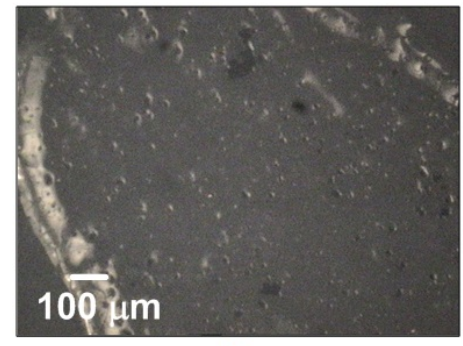

a

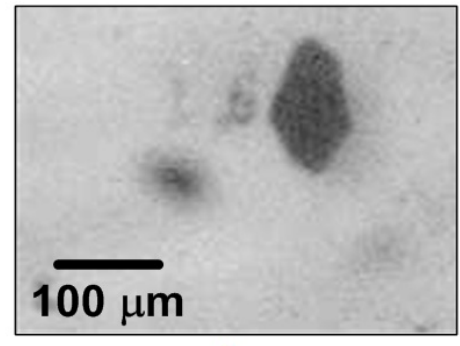

b

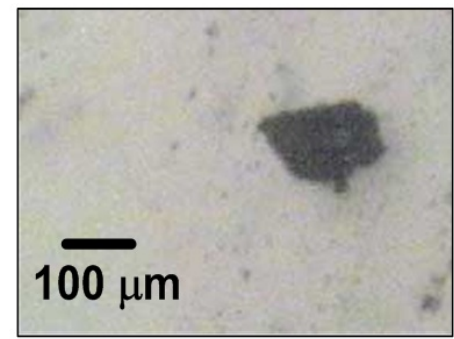

c

FIG. 1. Optical images of some RGO flakes on polystyrene matrix formed after precipitation. Black inclusions are RGO flakes

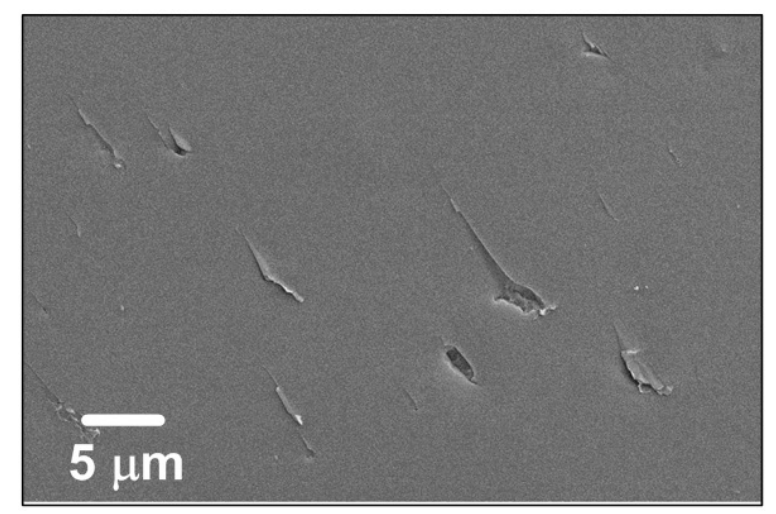

FIG. 2. SEM image of RGO distribution in polystyrene matrix without using precipitation [15]

Sufficiently large and round-shaped RGO flakes (Fig. 1b,c) were used for resistance measurements. In the case of steel electrodes with $15 \mu \mathrm{m}$ curvature radii, the resistances were measured for the large RGO flakes with diameter of $150-200 \mu \mathrm{m}$. Resistance of RGO flakes was dependent on the distance between measuring electrodes. The distance increase caused sharp resistance amplification. Resistances were evaluated from currentvoltage characteristics (Fig. 3,4). The resistance of RGO flakes was approximately $23 \mathrm{k} \Omega$ when distance between SPM tip electrodes was $10 \mu \mathrm{m}$ and $26 \mathrm{M} \Omega$ already for $30 \mu \mathrm{m}$ distance (Fig. 3). Resistance was $2 \Omega$ when measured by steel electrodes with larger curvature radii for $10 \mu \mathrm{m}$ gap and $5 \mathrm{k} \Omega$ for $30 \mu \mathrm{m}$ gap (Fig. 4). RGO 


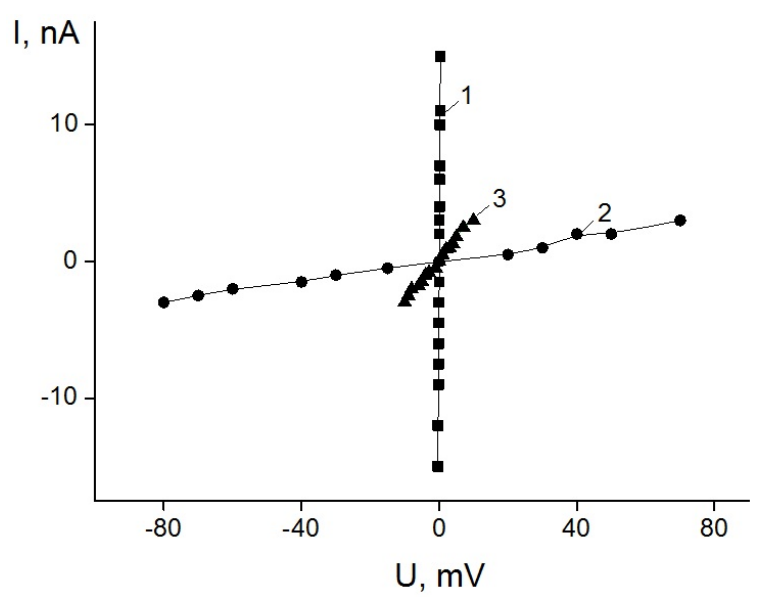

FIG. 3. Current-voltage characteristics of RGO flakes on polystyrene matrix using electrodes with $50 \mathrm{~nm}$ curvature radii $(1$ - distance between electrodes $10 \mu \mathrm{m}, 2$ - distance between electrodes $30 \mu \mathrm{m})$, and copper (3)

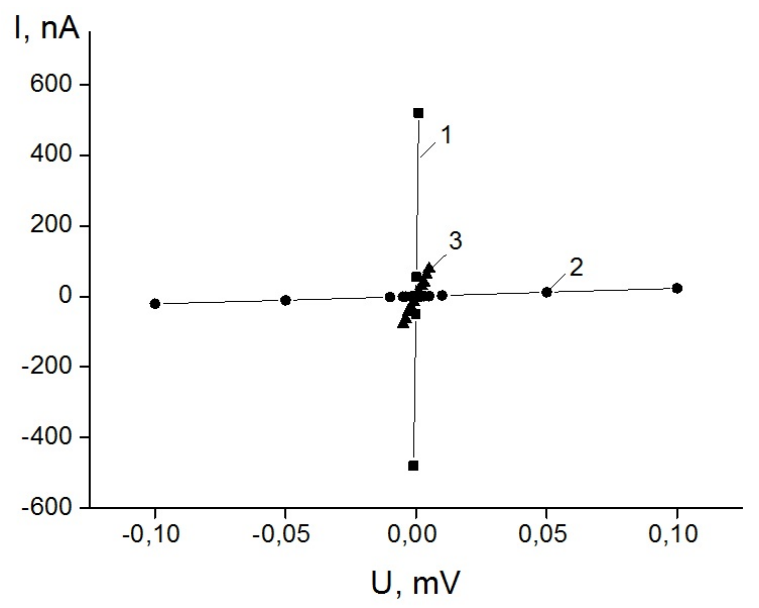

FIG. 4. Current-voltage characteristics of RGO flakes on polystyrene matrix using electrodes with $15 \mu \mathrm{m}$ curvature radii $(1-$ distance between electrodes $10 \mu \mathrm{m}, 2$ - distance between electrodes $30 \mu \mathrm{m})$, and copper (3)

flakes demonstrated non-conductive state for every kind of electrodes at a distance of $40 \mu \mathrm{m}$ and larger. It can be explained by defects number accumulation at the RGO surface with distance increase. The resistance of copper substrate was approximately $3.3 \mathrm{M} \Omega$ for tip and $63 \Omega$ for needle electrodes correspondingly (curve 3 on Fig. 3 and Fig. 4).

Ohm's nature of obtained current-voltage characteristics exclude the presence of a breakdown in films which have been investigated.

It should be noted that current values without voltage applying for $10 \mu \mathrm{m}$ distances between electrodes were approximately $1.5-2 \mathrm{nA}$. This fact is additional evidence of persistent currents in the RGO flakes. At larger distances between electrodes, the superconducting state disappears, so lines of current-voltage characteristics cross the zero point directly.

The obtained results of persistent currents observed at zero voltage can be explained by existence of superconducting phase in the RGO flakes at room temperature. Therefore the superconducting contribution in the resistance values is preserved in planar RGO structures at distances not exceeding $10 \mu \mathrm{m}$. We can suppose these multilayered RGO flakes obtained as a result of self-organization during precipitation process of RGO/polysterene composite are similar to finely dispersed pyrolytic graphite with superconducting inclusions [3]. All obtained results are in agreement with work [12], where it was shown for this RGO/polysterene composite that Josephson oscillations and Shapiro steps still exist up to room temperature. 


\section{Conclusions}

Large areas of planar structures from RGO flakes up to $100-200 \mu \mathrm{m}$ were obtained on polystyrene surface. For this purpose, precipitation of reduced graphene oxide composite on base of polystyrene from benzene was used. Precipitation was performed in 1:1 petroleum ether-benzene. The resistance of such planar inclusions, which was measured by diamond SPM tips with curvature of radii $50 \mathrm{~nm}$, turned out to be 2 orders of magnitude lower than the resistance measured for the copper substrate. A similar dependence for resistance was obtained using steel electrodes having curvature radii $15 \mu \mathrm{m}$.

\section{References}

[1] Ludbrook B.M., Levy G., Nigge P., et al. Evidence for superconductivity in Li-decorated monolayer graphene. Proc. Natl Acad. Sci. USA, 2015, 112(38), P. 11795-11799.

[2] Chapman J., Su Y., Howard C.A., et al. Superconductivity in Ca-doped graphene laminates. Sci. Rep., 2016 , 6, P. 23254.

[3] Saad M., Gilmutdinov I.F., Kiiamov A.G., et al. Observation of Persistent Currents in Finely Dispersed Pyrolytic Graphite. JETP Letters, 2018, 107(1), P. 37-41.

[4] Tonnoir C., Kimouche A., Coraux J., et al. Induced superconductivity in graphene grown on Rhenium. Phys. Rev. Lett., 2013, 111(24), P. 246805.

[5] Di Bernardo A., Millo O., Barbone M., et al. Corrigendum: p-wave triggered superconductivity in single-layer graphene on an electrondoped oxide superconductor. Nature Communications, 2017, 8, P. 14024.

[6] Esquinazi P., Heikkilä T.T., Lysogorskiy Y.V., et.al. On the superconductivity of graphite interfaces. JETP Letters, $2014,100(5)$, P. $336-339$.

[7] Uchoa B., Castro Neto A.H. Superconducting states of pure and doped graphene. Phys Rev Letters, 2007, 98, P. 146801.

[8] Lebedev S.G. Evidence of josephson-like behaviour of thin granular carbon films. International Review of Physics, 2008, 2(5), P. 312-328.

[9] Scheike T., Böhlmann W., Esquinazi P., et. al. Can doping graphite trigger room temperature superconductivity? Evidence for granular high-temperature superconductivity in water-treated graphite powder. Advanced Materials, 2012, 24(43), P. 5826-5831.

[10] Felner I., Kopelevich Y. Magnetization measurement of a possible high-temperature superconducting state in amorphous carbon doped with sulfur. Phys. Rev. B, 2009, 79(23), P. 233409.

[11] Ballestar A., Barzola-Quiquia J., Scheike T., et. al. Josephson-coupled superconducting regions embedded at the interfaces of highly oriented pyrolytic graphite. New J. Phys., 2013, 15(5), P. 023024.

[12] Ionov A.N. Josephson-Like Behaviour of the Current-Voltage Characteristics of Multi-graphene Flakes Embedded in Polystyrene. J. Low Temp. Phys., 2016, 185(5-6), P. 515-521.

[13] Volovik G.E., Pudalov V.M. Graphite on graphite. JETP Letters, 2016, 104(12), P. 880-882.

[14] Khairullin A.R., Nikolaeva M.N., Bugrov A.N. Resistance of the composite films based on polystyrene and graphene oxide. Nanosystems: physics, chemistry, mathematics, 2016, 7(6), P. 1055-1058.

[15] Nikolaeva M.N., Bugrov A.N., Anan'eva T.D., et al. Conductive properties of the composite films of graphene oxide based on polystyrene in a metal-polymer-metal structure. Russ. J. Appl. Chem., 2014, 87(8), P. 1151-1155.

[16] Yevlampieva N., Bugrov A., Anan'eva T., et al. Soluble poly (methyl methacrylate) composites containing covalently associated zirconium dioxide nanocrystals. Am. J. Nano Res. and Appl., 2014, 2(2), P. 1-8.

[17] Nikolaeva M.N., Anan'eva T.D., Bugrov A.N. et.al. Correlation between structure and resistance of composites based on polystyrene and multilayered graphene oxide. Nanosystems: physics, chemistry, mathematics, 2017, 8(2), P. 266-271.

[18] Nikolaeva M.N., Gushchina E V., Dunaevskii M.S., et al. The influence of substrate material on the resistance of composite films based on reduced graphene oxide and polystyrene. Nanosystems: physics, chemistry, mathematics, 2017, 8(5), P. $665-669$.

[19] Bugrov A.N., Zavialova A.Yu., Smyslov R.Yu., et al. Luminescence of $\mathrm{Eu}^{3+}$ ions in hybrid polymer-inorganic composites based on poly(methyl methacrylate) and zirconia nanoparticles. Journal of bioluminescence and chemiluminescence, 2018, P. 1-13. 